\title{
Adjuvant therapy for retroperitoneal sarcoma: a meta-analysis
}

\author{
Xiangji Li $\mathrm{Li}^{1,4}$, Tong $\mathrm{Wu}^{2}$, Mengmeng Xiao ${ }^{1}$, Shanshan $\mathrm{Wu}^{3}$, Li Min ${ }^{4}$ and Chenghua Luo ${ }^{1 *}$ (D)
}

\begin{abstract}
Background: Adjuvant therapy is a promising treatment to improve the prognosis of cancer patients, however, the evidence base driving recommendations for adjuvant radiotherapy (ART) or chemotherapy (ACT) in retroperitoneal sarcomas (RPS) primarily hinges on observational data. The aim of this study was to evaluate the effectiveness of adjuvant therapy in the management of RPS patients.

Methods: We searched PubMed, Web of Science, Embase, ASCO Abstracts, and Cochrane Library for comparative studies (until December 2020) of adjuvant therapy versus surgery alone. Data on the following endpoints were evaluated: overall survival (OS), local recurrence (LR), recurrence-free survival (RFS), and metastasis-free survival (MFS). Data were summarized as hazard ratios (HR) with 95\% confidence intervals (CI). Risk of bias of studies was assessed with Begg's and Egger's tests.

Results: A total of 15 trials were eligible, including 9281 adjuvant therapy and 21,583 surgery alone cases (20 studies for OS, six studies for RFS, two studies for LR, and two studies for MFS). Meta-analysis showed that ART was associated with distinct advantages as compared to surgery alone, including a longer OS ( $H R=0.80, P<0.0001)$, a longer RFS $(H R=0.61, P=0.0002)$, and a lower $L R(H R=0.31, P=0.005)$. However, this meta-analysis failed to demonstrate a benefit of ACT for RPS patients, including OS (HR=1.11, $P=0.19)$, RFS (HR=1.30, $P=0.09)$ and MFS (HR=0.69, $P=0.09$ ). In the sensitivity analysis, $A C T$ was associated with a worse $O S(H R=1.19, P=0.0002)$. No evidence of publication bias was observed.
\end{abstract}

Conclusions: Overall, the quality of the evidence was moderate for most outcomes. The evidence supports that ART achieved a generally better outcome as compared to surgery alone.

Keywords: Meta-analysis, Retroperitoneal sarcoma, Adjuvant therapy, Surgery

\section{Introduction}

Retroperitoneal sarcomas (RPS) are the second common malignancy after soft tissue sarcoma (STS) of the extremities, accounting for $10-15 \%$ of all STS and $30 \%$ of all malignant retroperitoneal tumors [1]. R0 surgical resection is the most potential treatment to cure patients with localized disease, which means the adjacent organs

\footnotetext{
${ }^{*}$ Correspondence: luochenghua@pkuih.edu.cn

1 Department of Retroperitoneal Tumor Surgery, Peking University International Hospital, 1ShengMingYuan Road, Beijing 102206, People's Republic of China

Full list of author information is available at the end of the article
}

invaded are often not preserved. According to current studies, the rate of complete resection ranges from 41.8 to $76 \%$ [2]. However, local recurrence remains high even if negative margins is being achieved as far as possible, and leads to poor prognosis, with 5-year overall survival (OS) ranging from 39 to $65 \%$ and a mortality rate of $20-75 \%$ [3-7].

To improve local control and overall survival, adjuvant therapy (AT) such as adjuvant radiotherapy (ART) has been investigated. However, there are insufficient evidences to compile treatment guidelines due to the different conclusions based on limited retrospective clinical studies (RCSs) [8-22]. For example, multiple analyses of original author(s) and the source, provide a link to the Creative Commons licence, and indicate if changes were made. The images or other third party material in this article are included in the article's Creative Commons licence, unless indicated otherwise in a credit line to the material. If material is not included in the article's Creative Commons licence and your intended use is not permitted by statutory regulation or exceeds the permitted use, you will need to obtain permission directly from the copyright holder. To view a copy of this licence, visit http://creativecommons.org/licenses/by/4.0/. The Creative Commons Public Domain Dedication waiver (http://creativeco mmons.org/publicdomain/zero/1.0/) applies to the data made available in this article, unless otherwise stated in a credit line to the data. 
the Surveillance, Epidemiology, and End Results (SEER) database and a retrospective analysis from French have shown that ART could not improve OS in RPS patients $[5,9,12,23]$, but Trovik et al. [8] and others [10, 18] demonstrated a significant improvement in OS as well as recurrence-free survival (RFS) in patients undergoing ART, which are obviously confusing. In addition, most trials were undertaken in the setting of advanced extremity sarcomas and the generalizability of these data is limited [24, 25], resulting chemotherapy cannot be as a standard approach to treat RPS. Therefore, it is greatly clinical significance to discuss whether advanced RPS patients can benefit from adjuvant chemotherapy (ACT).

The aim of this meta-analysis is to review the latest body of literature comparing AT with surgery alone in RPS patients, and to clarify the role of ART and ACT in the prognostic outcome of these patients.

\section{Methods}

\section{Searching strategy}

The meta-analysis was conducted according to the guidelines of the Cochrane Handbook, and reported according to the Preferred Reporting Items for Systematic Reviews and Meta-Analyses (PRISMA) statement. We search PubMed, Web of Science, Embase, ASCO Abstracts, and Cochrane library for eligible studies between January 2000 and December 2020 with the searching strategy: (((adjuvant radiotherapy OR postoperative radiotherapy OR postoperative chemotherapy) AND (retroperitoneal sarcomas OR retroperitoneal soft tissue sarcomas OR retroperitoneal neoplasms)) AND (surgery))). In addition, reference lists of all studies were screened to identify potentially eligible studies.

\section{Selection criteria}

Studies were included based on following criteria: 1) randomized clinical trial (RCT), case-control study, or retrospective cohort study of AT versus surgery for RPS patients; 2) PRS confirmed by pathological biopsy; 3) studies providing data of hazard ration (HR) and 95\% confidence interval (CI) of local recurrence (LR), metastasis-free survival (MFS), RFS or OS of AT versus surgery for RPS patients. The exclusion criteria included: (1) letter, editorial or noncomparative study; 2 ) the cases or the groups in the study were less than 20 and 5 respectively; (3) HR and 95\% CI cannot be extracted from studies; 4) non-human studies.

\section{Data extraction and quality assessment}

Two authors independently extracted data using a standard form from eligible studies. Discrepancies were resolved by consensus, and invited a third investigator to interpret if the differences remained controversial after discussion. The following information was extracted from each included study: primary author, year of publication, patient source, study type, number of patients, age, high malignancy grade, tumour size, intervention, surgical margins, dose of radiotherapy (drug of chemotherapy), and data of OS, LR, RFS and MFS. All included studies were assessed by Newcastle-Ottawa Scale (NOS). The assessment tool focused on three aspects includes participant selection, comparability and exposure with 9 items. A study was considered of high quality if it scored 7 points or higher.

\section{Statistical analysis}

Meta-analysis was performed by Review Manager version 5.4 (Cochrane Collaboration, London, UK). HR was used as a summary statistic and calculated using either fixed-effects models or, in the presence of heterogeneity $\left(P<0.10, I^{2}>50 \%\right)$, random-effects models. Sensitivity analysis was used to identify the possible sources of heterogeneity and detect the stability of studies by re-metaanalysis with one involved study excluded each time. Publication bias was assessed using Begg's and Egger's test with stataCorp version 15.1 (College Station, TX 77,845, USA). All P-values were two-sides.

\section{Results}

\section{Search results and characteristic of included studies}

The detailed characteristics of included studies and the results of the quality assessment are summarized in Table 1. A total of 2641 references were identified form databases of PubMed, Web of Science, Embase, ASCO Abstracts and Cochrane library. After selection according to the inclusion/exclusion criteria, 15 RCSs were eligible for meta-analysis (Fig. 1). In these studies, 30,864 patients with RPS were compared, including 9281 patients who underwent adjuvant therapy and 21,583 patients who underwent surgery alone. In addition, five studies performed concurrent ART versus surgery and ACT versus surgery, four studies divide into adjuvant therapy group and surgery group with propensity score marched (PSM). The earliest study was published in 2008 , and the latest in 2019. Studies were conducted in four different countries (USA, France, Norway and Italy). All studies were evaluated by NOS and the overall quality averaged 7.45 stars (range 7-8) on scale of 0-9 (Additional file 1: Table S1).

\section{Meta-analysis of OS}

The fixed-effects results showed that the OS was significantly improved in ART group as compared to surgery alone group $(\mathrm{HR}=0.80,95 \%$ CI $0.76-0.84 ; P<0.0001$ ) (Fig. 2a). However, there was no significant difference in ACT group versus surgery alone group ( $\mathrm{HR}=1.11,95 \%$ CI $0.95-1.29 ; P=0.19$ ) (Fig. 3a). Notable heterogeneity 


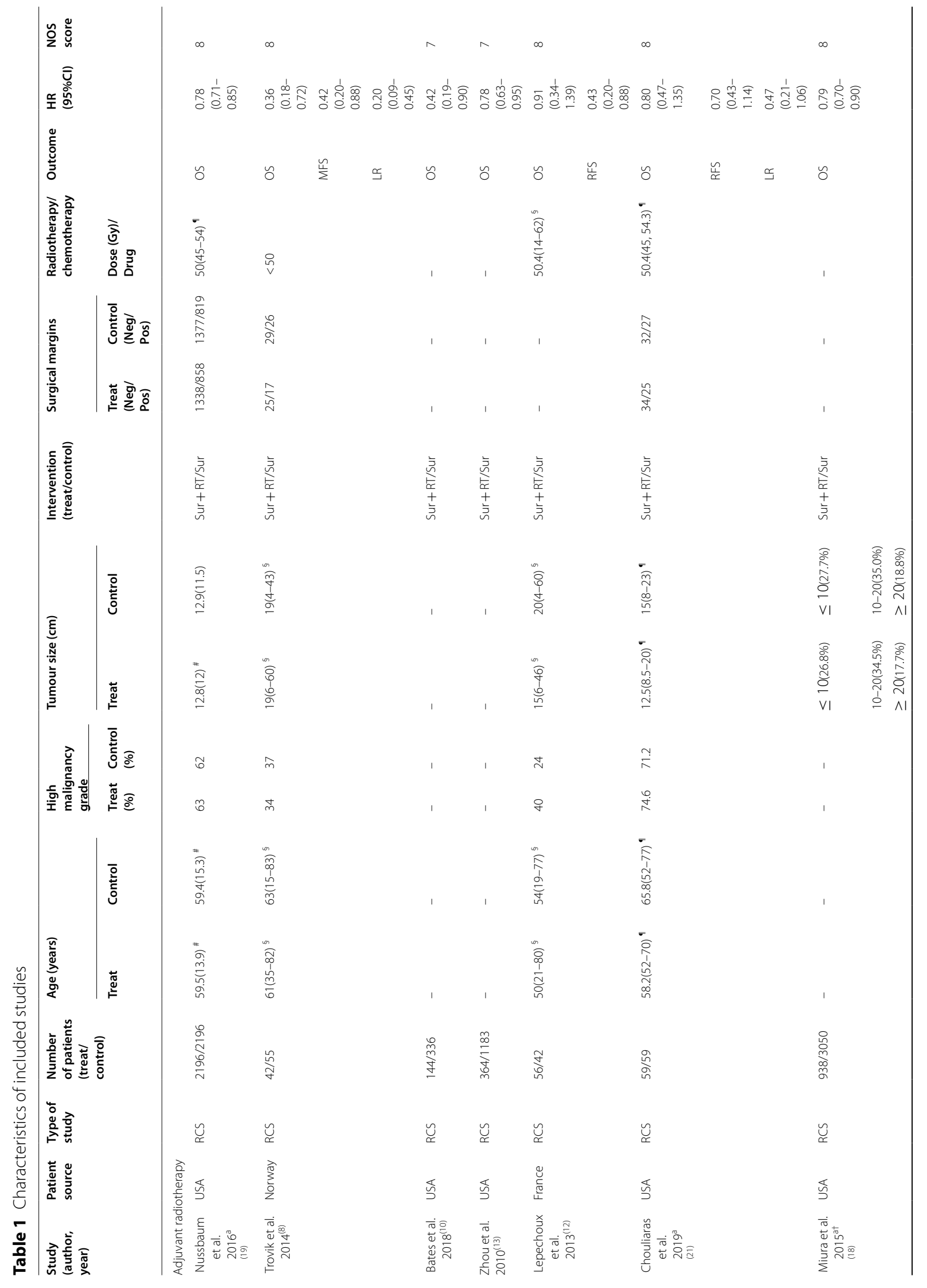




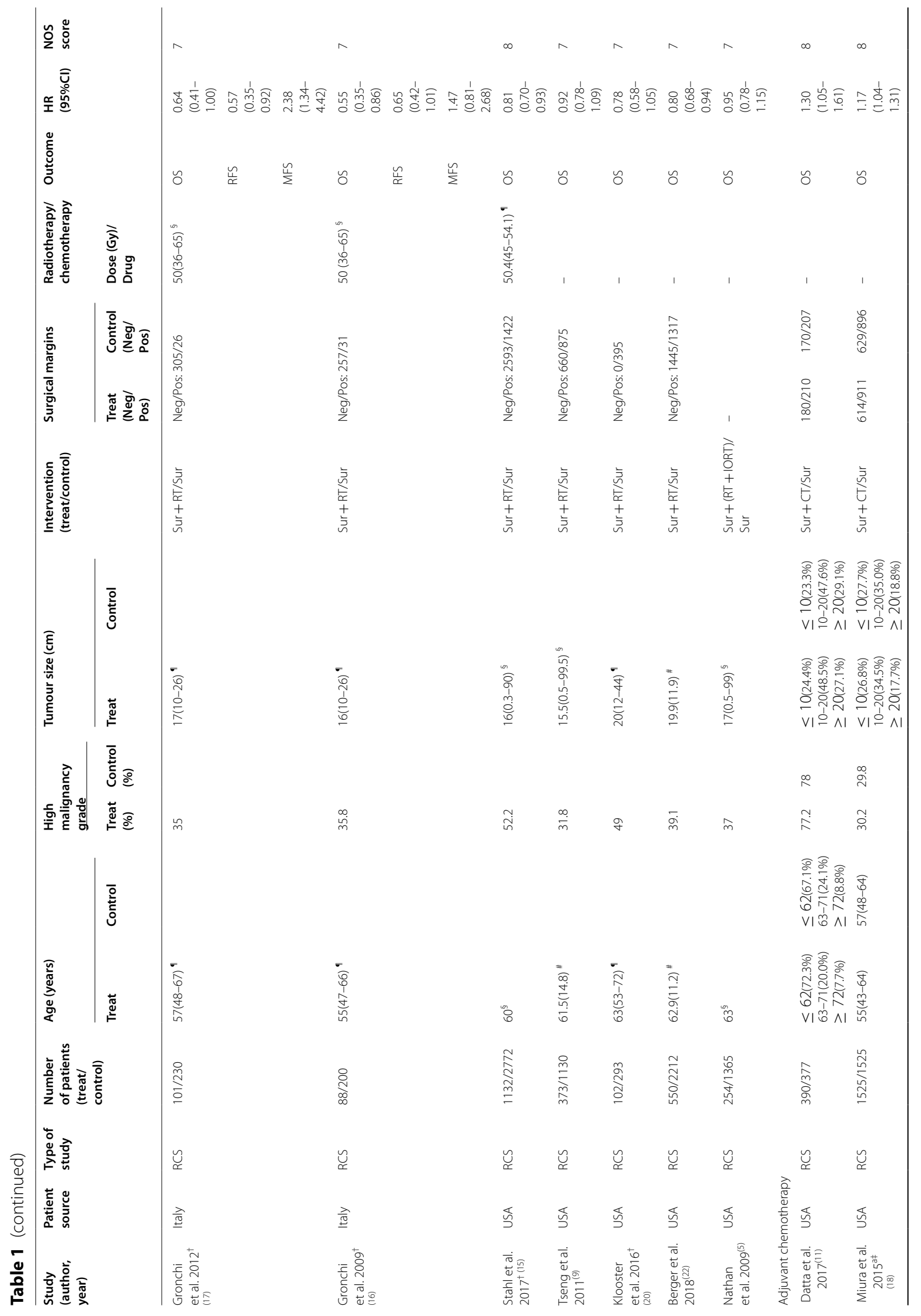




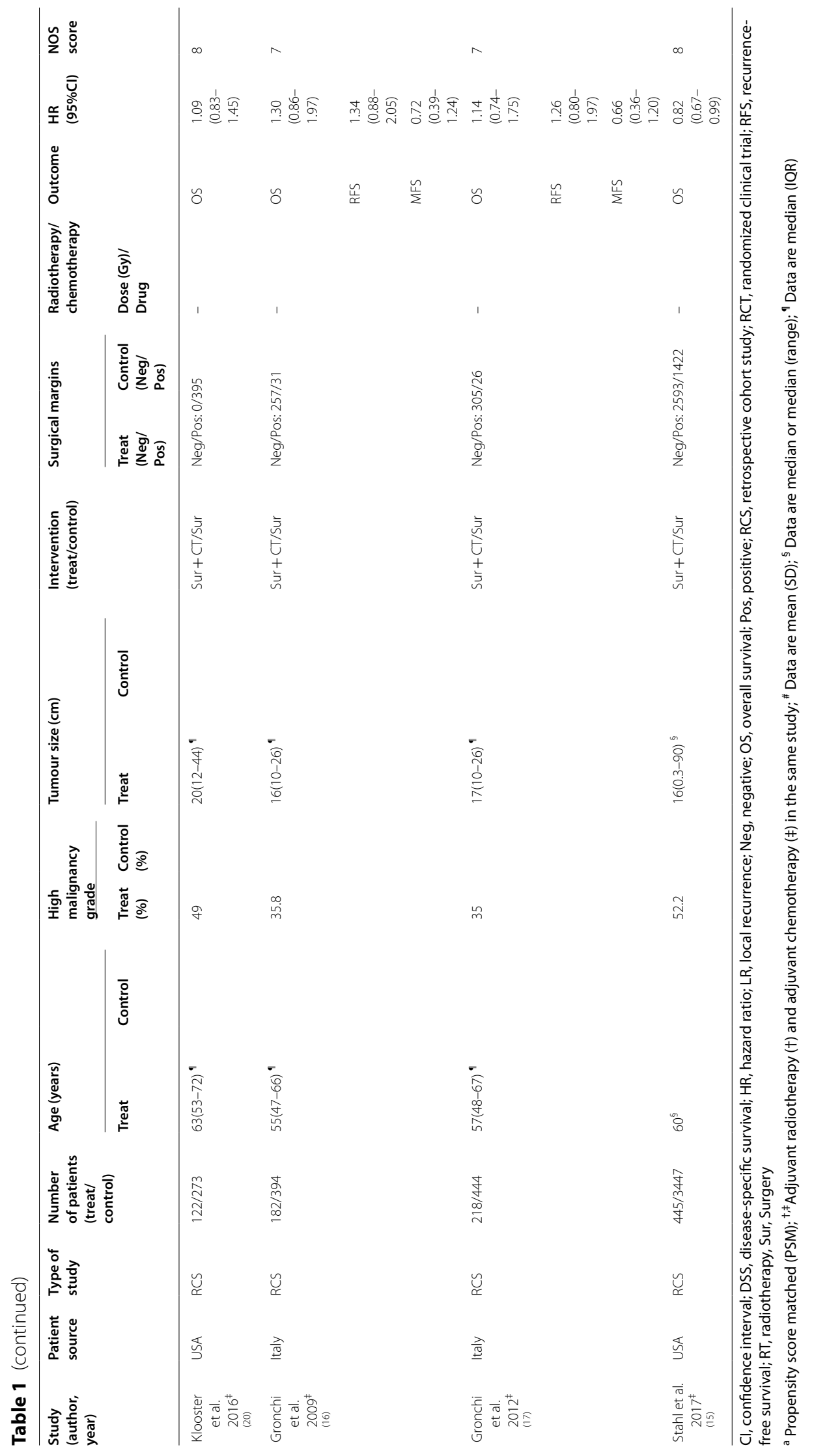


was seen in the latter, and the sensitivity analysis indicated that patients benefited more from surgery alone than ACT $(\mathrm{HR}=1.19,95 \%$ CI 1.08-1.30; $P=0.0002)$.

\section{Meta-analysis of RFS}

The meta-analysis in the fixed-effects model indicated that RFS was obviously improved in ART group $(\mathrm{HR}=0.61,95 \% \mathrm{CI}: 0.47-0.79 ; P=0.0002)$, and the four sets of results showed no significant amount of heterogeneity (Fig. 2b). However, there was no significant difference in ACT versus surgery $(\mathrm{HR}=1.30,95 \% \mathrm{CI}$ $0.96-1.77 ; P=0.09$ ), no statistical heterogeneity was found (Fig. 3b).

\section{Meta-analysis of LR}

LR was reported in two PSM studies, including 101 patients in ART group and 114 patients in surgery group. The result showed LR of ART group was much lower than surgery group $(\mathrm{HR}=0.31,95 \% \mathrm{CI} 0.13-0.71$; $P=0.005)$ (Fig. 2c).

\section{Meta-analysis of MFS}

The MFS was reported by two studies in ACT versus surgery, 1238 participants. There was no statistical significance between the two comparisons ( $\mathrm{HR}=0.69,95 \%$ CI $0.45-1.06 ; P=0.09$ ), no statistical heterogeneity was found (Fig. 3c).

\section{Publication bias}

The detailed results of pooled analysis and the heterogeneity analysis are summarized in Table 2 . Publication bias was determined by Begg's and Egger's tests, and there was no evidence of publication bias for OS and RFS (Fig. 4a-c and Additional file 1: Table S2). However, there were significant heterogeneity in two pooled analysis, sensitivity analysis showed that one of which had a different result after excluding the most heterogeneous study. We will analyze this in the later part of discussion.

\section{Discussion}

The unique biological behavior of RPS brings great challenges to clinicians in the treatment of this disease. R0 resection is the only potentially curative therapy, but the risk of LR is substantial because large size and anatomical structure of the tumor frequently preclude resection with widely clear margins. Five-year LR rates range from 28 to $60 \%$ [16, 26-28]. With long-term follow-up, almost all patients are likely to recur [29]. Therefore, it is urgent to clarify the comprehensive treatment of RPS. ART, remains a controversial component of treatment, unlike STS in extremity, there is no rigorous level I data to support ART significantly reduce LR in RPS patients, and lessons from ART trials in extremity STS are difficult to

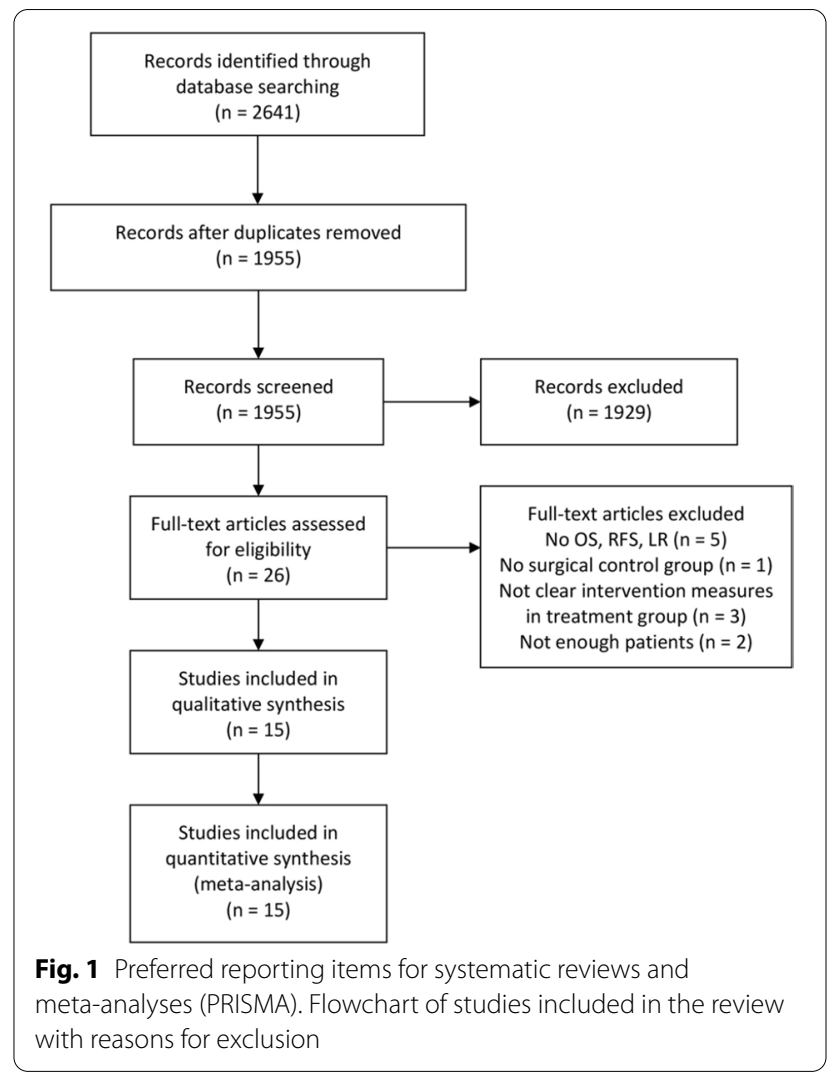

translate directly to the RPS due to the potential for significant toxicity at equivalent dose [26]. Here our pooled analysis revealed that LR of RPS patients is distinctly reduce in ART group as compared to surgery alone, it is a key parameter to evaluate the prognosis of patients under different treatments. Besides, whether ART is beneficial to OS is also controversial. Several RCSs indicated consistently that ART improve local control (LC) and RFS but failed to demonstrate a statistically significant association with OS $[5,9,12,21]$. Others obtained opposite results that ART not only improved the OS, but also has obvious curative effect in RFS and LC with a premise that the margin of surgical resection reaches $\mathrm{R} 0$ or $\mathrm{R} 1[3,10$, $11,13,16,18]$. In our meta-analysis, the results support the latter.

[In addition to therapeutic effects, radiation toxicities are primary consideration in selecting an appropriate radiation dose. Among the included studies, detailed radiation doses were given in seven studies (Table 1 ), and the median radiation doses were all $50 \mathrm{~Gy}$, with the highest not exceeding $65 \mathrm{~Gy}$. However, radiotherapy toxicities and target volumes were not mentioned in any of these studies, and the main reason was the lack of records on the aspect in the databases selected by these retrospective studies. Besides, the location occupied by the original 


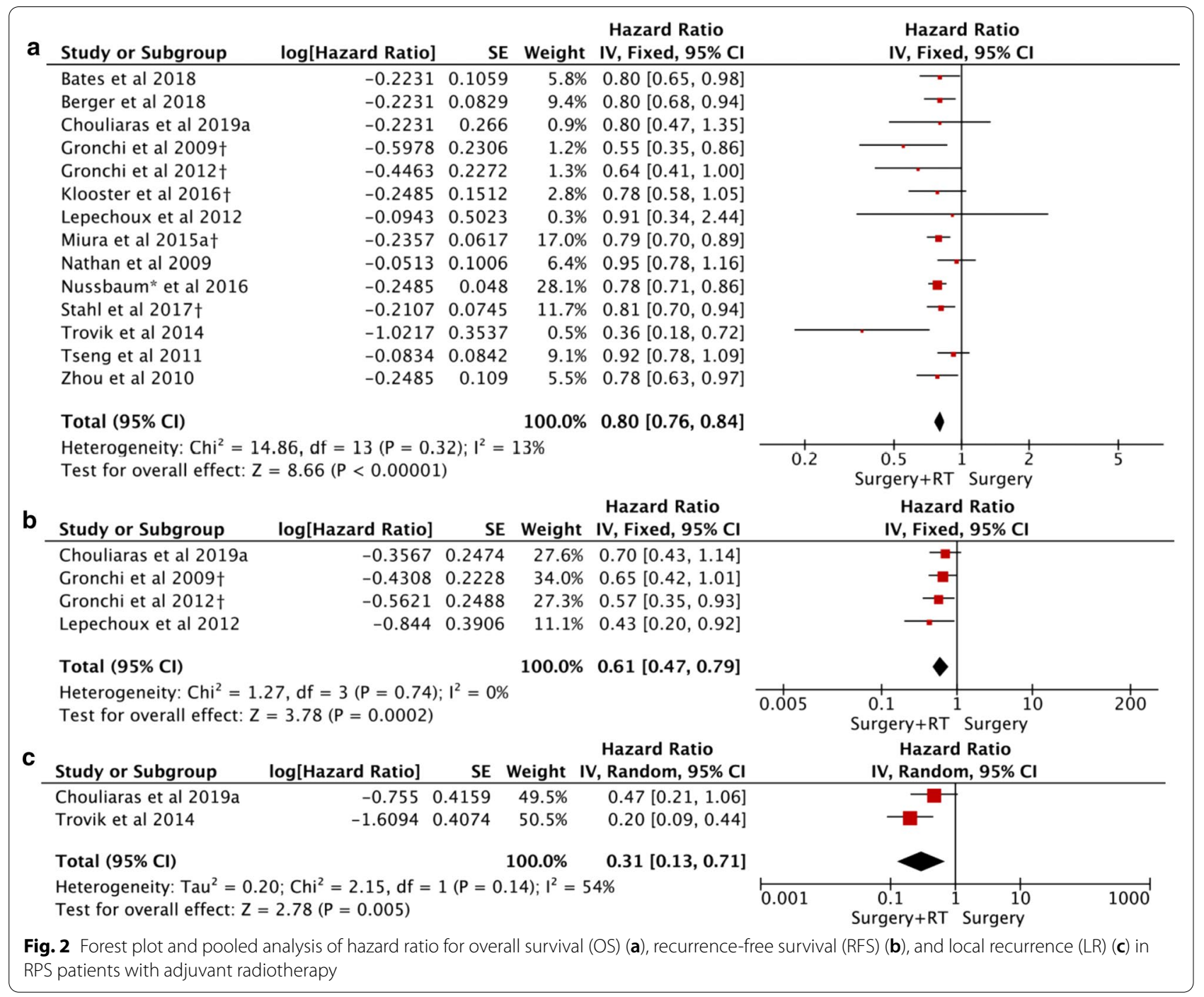

sarcomas would be filled with adjacent normal tissues after resection, which also led to uncertainty in the radiotherapy target volumes. Previous two trials (phase1 and phase 2) have been reported that 45-54 Gy were optimal doses according to the potential benefits and risks assessed by them, to avoid bowel complications reported with higher doses and the potential negative impact on surgery [30, 31]. Recently, a phase 3 trials (multicenter, open-label, randomized) analysed the radiation toxicities in the ART, radiotherapy was delivered as 50.4 Gy (in 28 daily fractions of $1.8 \mathrm{~Gy}$ ). The resulted showed the most common grade 3-4 adverse events (lymphopenia, anaemia, and hypoalbuminaemia) were reported in 127/127 patients in ART group, and in 16/128 patients in surgery alone. Serious adverse events were reported in 30/127 patients in ART group, and in 13/128 patients in surgery alone. One patient was died in ART group due to gastropleural fistula [32]. Complications rates were lower in this trial than have been reported with ART, ranging from20$40 \%$ in retrospective series $[12,33,34]$. In our study, the radiation doses performed were basically consistent with the optimal doses Therefore, we considered that the radiation toxicities are acceptable, but the specific radiation toxicities need to be further investigated.]

Anthracyclines were the first systemic chemotherapeutic agents to demonstrate activity in STS, and doxorubicin was main representative. According currently studies, using ACT in STS could not benefit patients, neither patients whose tumors remained resectable or patients who had metastasized at an advanced stage. A multicenter phase III RCT (EORTC) [35] randomly assigned 351 patients with non-metastatic macroscopically resected II-III tumors to the postoperative chemotherapy group (175 patients with ifosfamide/ doxorubicin) or to the control group (176 patients). The results demonstrated OS did not differ significantly 


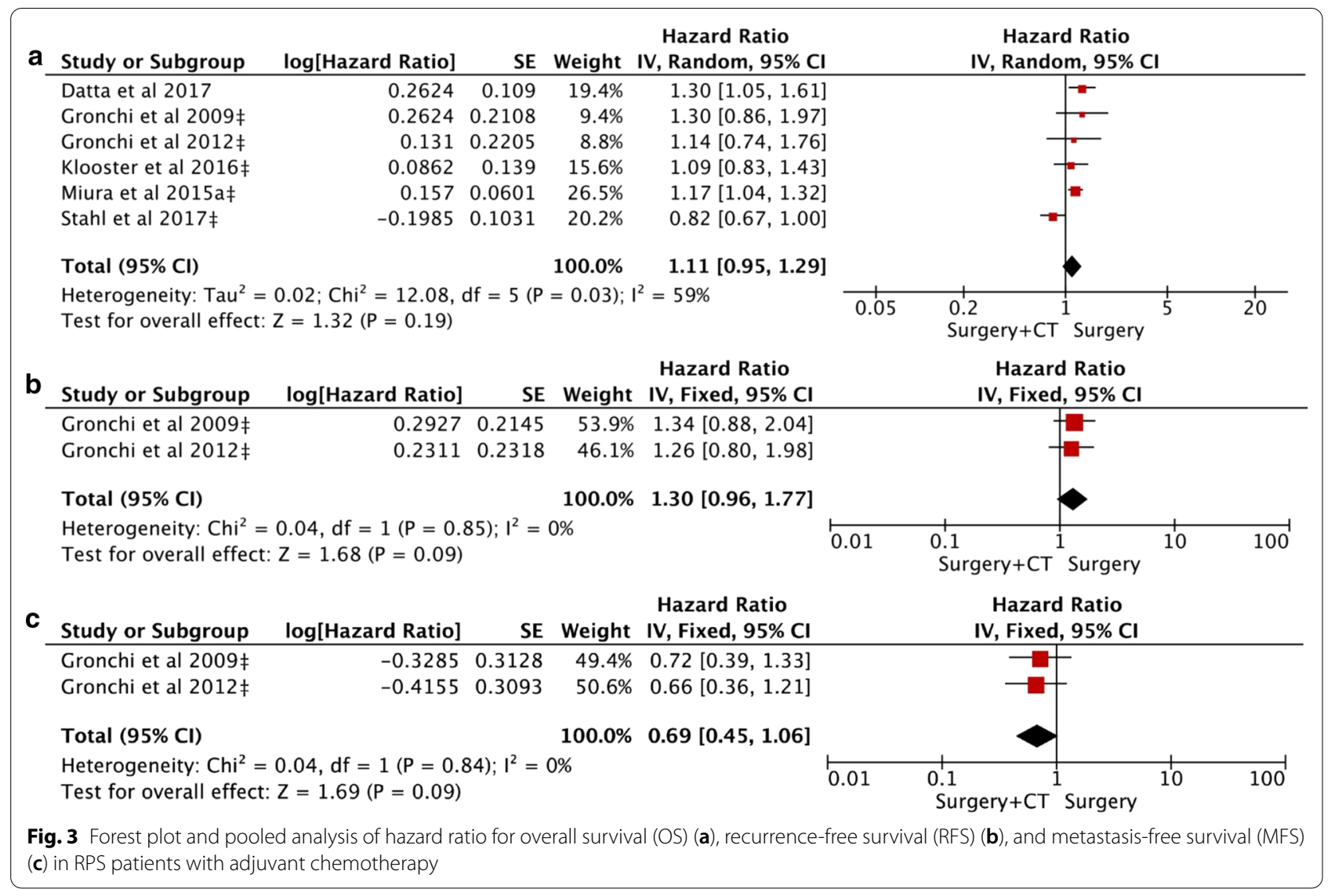

Table 2 Summary of results

\begin{tabular}{|c|c|c|c|c|c|c|c|c|c|}
\hline \multirow[t]{2}{*}{ Categories } & \multirow[t]{2}{*}{ Studies } & \multirow[t]{2}{*}{ Patients } & \multirow[t]{2}{*}{ Model } & \multicolumn{3}{|l|}{$\mathrm{HR}(95 \% \mathrm{Cl})$} & \multicolumn{3}{|c|}{ Heterogeneity } \\
\hline & & & & value & $z$ & P-value & $\mathrm{x}^{2}$ & $I^{2}$ & P-value \\
\hline \multicolumn{10}{|l|}{ OS } \\
\hline Adjuvant radiotherapy & 14 & 20,564 & Fixed & $0.80(0.76-0.84)$ & 8.66 & $<0.0001$ & 14.86 & $13 \%$ & 0.32 \\
\hline Adjuvant chemotherapy & 6 & 9342 & Random & $1.11(0.95-1.29)$ & 1.32 & 0.19 & 12.08 & $59 \%$ & 0.03 \\
\hline $\begin{array}{l}\text { Sensitivity analysis of adju- } \\
\text { vant chemotherapy }\end{array}$ & 5 & 5450 & Fixed & $1.19(1.08-1.30)$ & 3.68 & 0.0002 & 1.35 & $0 \%$ & 0.85 \\
\hline \multicolumn{10}{|l|}{ RFS } \\
\hline Adjuvant radiotherapy & 4 & 1454 & Fixed & $0.61(0.47-0.79)$ & 3.78 & 0.0002 & 1.27 & $0 \%$ & 0.74 \\
\hline Adjuvant chemotherapy & 2 & 1238 & Fixed & $1.30(0.96-1.77)$ & 1.68 & 0.09 & 0.04 & $0 \%$ & 0.85 \\
\hline \multicolumn{10}{|l|}{ LR } \\
\hline Adjuvant radiotherapy & 2 & 215 & Random & $0.31(0.13-0.71)$ & 2.78 & 0.005 & 2.78 & $54 \%$ & 0.14 \\
\hline \multicolumn{10}{|l|}{ MFS } \\
\hline Adjuvant chemotherapy & 2 & 1238 & Fixed & $0.69(0.45-1.06)$ & 1.69 & 0.09 & 0.04 & $0 \%$ & 0.84 \\
\hline
\end{tabular}

$\mathrm{Cl}$, confidence interval; $\mathrm{HR}$, hazard ratio; LR, local recurrence; OS, overall survival; RFS, recurrence-free survival

between groups $(\mathrm{HR}=0.94,95 \%$ CI $0.68-1.31 ; P=0.72)$ nor did RFS (HR $=0.91,95 \%$ CI $0.67-1.22 ; P=0.51)$. A retrospective study of efficiency of ACT in resected RPS [11], published in 2017, showed that utilization of ACT was associated with worse long-term survival
(HR $=1.30,95 \%$ CI 1.05-1.61; $P=0.017)$. However, the trend of OS improvement with ACT were found in spindle cell $(\mathrm{HR}=0.37,95 \% \mathrm{CI} 0.10-1.38)$, giant cell $(\mathrm{HR}=0.82,95 \% \mathrm{CI} 0.32-2.13)$ and synovial sarcoma $(\mathrm{HR}=0.26,95 \%$ CI $0.05-1.33)$. Here our findings show 

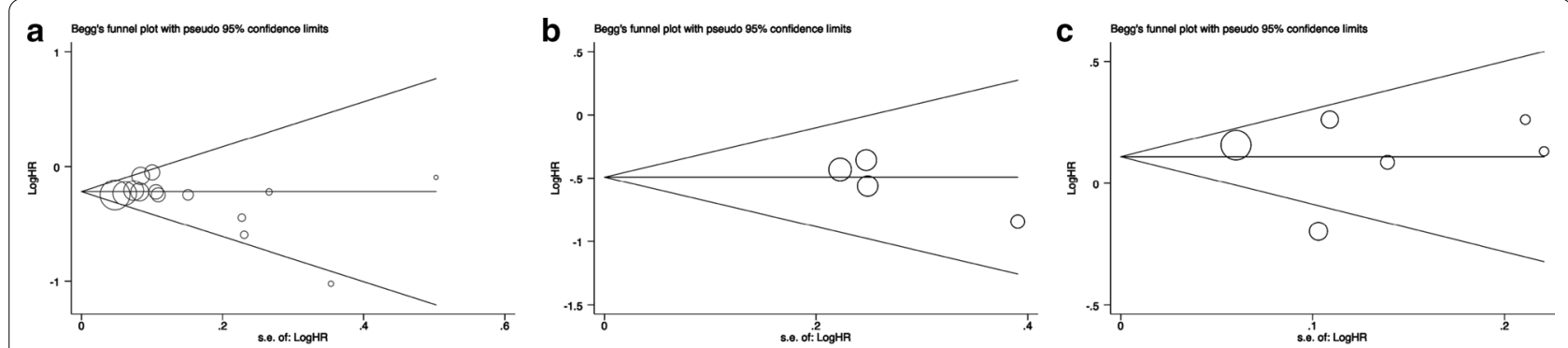

Fig. 4 Begg's test for overall survival (OS) and recurrence-free survival (RFS) in RPS patients with adjuvant radiotherapy (a, b), and overall survival (OS) in RPS patients with adjuvant chemotherapy (c)

that ACT cannot benefit RPS patients from OS, RFS and MFS, and even as previously reported study, it may cause worse OS (sensitivity analysis). Although whether metastasis occurs for patients is not the main reason to cause the increase in mortality, MFS was also included in the study as a prognostic parameter for PRS. In the studies we reviewed, only two studies included MFS $[16,17]$, and the two studies were conducted by the same author in different years.

In our study, notable heterogeneity was seen in LR of ART and OS of ACT, and only OS can perform sensitivity analysis due to lack of studies in LR. The sensitivity analysis showed that ACT was associated with worse OS, which was different from previous results of pooled analysis. In any case, the two results support that ACT cannot improve OS in patients with RPS. From another perspective, we have to admit that the result is not robust. Meanwhile, some limitations should be considered before appraising the results of this study. First, interventions in the treatment group in some studies were not limited to postoperative radiotherapy and chemotherapy, and in order to minimize the interference factors, we extracted HR form the multivariate COX regression analysis. Second, all included studies were RCSs, no RCT was found in databases we search. Finally, studies were insufficient in the sub-analysis of LR of ART and RFS of ACT duo to only two studies were included, limiting the validity of the comparisons between studies and conclusions drawn.

In this study, the quality of evidence was moderate but sufficient to establish the efficacy of ART for RPS. The relationship between ACT and the prognosis of RPS needs to be further studied, especially for patients with resectable RPS. Since there was a trend that ART is more likely to improve OS and LC of RPS patients, while $\mathrm{ACT}$ is for MFS, distinguishing the different efficiency between ART and ACT was also urgent. In addition, further studies could significantly change the results that ACT was associated with a wore OS, and more prognostic factors, such as pathological type, surgical resection method, dose, and related toxic complications, need to be included for analysis. Of course, all results will eventually need to be verified by multicentered RCTs.

\section{Conclusions}

Overall, our Meta-analysis showed RPS patients who underwent ART had better prognostic outcome than those who underwent surgery alone, including a longer OS, a longer RFS, and a lower LR. However, Those positive therapeutic effects have not been demonstrated in $\mathrm{ACT}$, either in OS, RFS or MFS.

\begin{abstract}
Abbreviations
ART: adjuvant radiotherapy; ACT: adjuvant chemotherapy; RPS: retroperitoneal sarcomas; OS: overall survival; LR: local recurrence; RFS: recurrence-free survival; MFS: metastasis-free survival; HR: hazard ratios; Cl: confidence intervals; AT: adjuvant therapy; STS: soft tissue sarcoma; RCS: retrospective clinical study; SEER: surveillance, epidemiology, and end results; PRISMA: preferred reporting items for systematic reviews and meta-analyses; RCT: randomized clinical trial; NOS: Newcastle-Ottawa scale (NOS); PSM: propensity score marched; LC: local control.
\end{abstract}

\section{Supplementary information}

The online version contains supplementary material available at https://doi. org/10.1186/s13014-021-01774-w.

Additional file 1. The Quality of Enrolled Studies, and The Publication Bias of Subgroup Analysis. Table S1. The Quality of Enrolled Studies. Table S2. Begg's and Egger's Test for Publication Bias.

\section{Acknowledgements}

The authors would like to acknowledge Ferdinando Carlo Maria Cananzi, MD, Vittorio Quagliuolo, MD (Department of Biomedical Sciences, Humanitas University), and Ruihan Dong, MBBS (Beijing Shijitan Hospital, Capital Medical University), for helpful discussions. The contributions were not compensated.

\section{Authors' contributions}

$\mathrm{XL}, \mathrm{TW}$, and $\mathrm{CL}$ contributed substantially to the conception, design and acquisition of data. $\mathrm{XL}, \mathrm{LM}$, and TW wrote the main manuscript text. $\mathrm{XL}$, TW, $L M$, and MX prepared figures. XL, SW, MX, and TW contributed to the analysis and interpretation of the data. $\mathrm{X}, \mathrm{SW}, \mathrm{LM}$, and $\mathrm{CL}$ contributed to devising the draft of the article and all of the authors revised it critically. All authors participated in revising the manuscript and in the final approval of the version to be published. All authors read and approved the final manuscript. 


\section{Funding}

This study was funded by Peking University International Hospital Research Grant (YN2019QN11); Beijing science and technology commission "Applied research on clinical characteristics of the capital city" (Z171100001017095).

\section{Availability of data and materials}

The datasets used and/or analyzed during the current study are available from the corresponding author on reasonable request.

\section{Declarations}

Ethic approval and consent to participate

Not applicable.

\section{Competing interests}

The authors declare that they have no conflict of interests.

\begin{abstract}
Author details
1 Department of Retroperitoneal Tumor Surgery, Peking University International Hospital, 1ShengMingYuan Road, Beijing 102206, People's Republic of China. ${ }^{2}$ Department of Sports Medicine, Peking University Third Hospital, Institute of Sports Medicine of Peking University, Beijing Key Laboratory of Sports Injuries, Beijing, People's Republic of China. ${ }^{3}$ Clinical Epidemiology and EBM Unit, National Clinical Research Center for Digestive Disease, Beijing Friendship Hospital, Capital Medical University, Beijing, People's Republic of China. ${ }^{4}$ Department of Gastroenterology, National Clinical Research Center for Digestive Disease, Beijing Digestive Disease Center, Beijing Key Laboratory for Precancerous Lesion of Digestive Disease, Beijing Friendship Hospital, Capital Medical University, Beijing, People's Republic of China.
\end{abstract}

Received: 14 December 2020 Accepted: 23 February 2021 Published online: 07 October 2021

\section{References}

1. Porter GA, Baxter NN, Pisters PW. Retroperitoneal sarcoma: a populationbased analysis of epidemiology, surgery, and radiotherapy. Cancer. 2006;106(7):1610-6.

2. Toulmonde M, Bonvalot S, Méeus P, et al. Retroperitoneal sarcomas: patterns of care at diagnosis, prognostic factors and focus on main histological subtypes: a multicenter analysis of the French Sarcoma Group. Ann Oncol. 2014;25(3):735-42.

3. Bonvalot $\mathrm{S}$, Miceli $\mathrm{R}$, Berselli $\mathrm{M}$, et al. Aggressive surgery in retroperitoneal soft tissue sarcoma carried out at high-volume centers is safe and is associated with improved local control. Ann Surg Oncol. 2010;17(6):1507-14.

4. Gronchi A, Miceli R, Shurell E, et al. Outcome prediction in primary resected retroperitoneal soft tissue sarcoma: histology-specific overall survival and disease-free survival nomograms built on major sarcoma center data sets. J Clin Oncol. 2013;31(13):1649-55.

5. Nathan $\mathrm{H}$, Raut CP, Thornton K, et al. Predictors of survival after resection of retroperitoneal sarcoma: a population-based analysis and critical appraisal of the AJCC staging system. Ann Surg. 2009;250(6):970-6.

6. van Dalen T, Plooij JM, van Coevorden F, et al. Long-term prognosis of primary retroperitoneal soft tissue sarcoma. Eur J Surg Oncol. 2007;33(2):234-8.

7. Van De Voorde L, Delrue L, van Eijkeren M, et al. Radiotherapy and surgery-an indispensable duo in the treatment of retroperitoneal sarcoma. Cancer. 2011;117(19):4355-64.

8. Trovik LH, Ovrebo K, Almquist M, et al. Adjuvant radiotherapy in retroperitoneal sarcomas. A Scandinavian Sarcoma Group study of 97 patients. Acta Oncol. 2014;53(9):1165-72.

9. Tseng WH, Martinez SR, Do L, et al. Lack of survival benefit following adjuvant radiation in patients with retroperitoneal sarcoma: a SEER analysis. J Surg Res. 2011;168(2):e173-80.

10. Bates JE, Dhakal S, Mazloom A, et al. The benefit of adjuvant radiotherapy in high-grade nonmetastatic retroperitoneal soft tissue sarcoma: a SEER analysis. Am J Clin Oncol. 2018:41(3):274-9.

11. Datta J, Ecker BL, Neuwirth MG, et al. Contemporary reappraisal of the efficacy of adjuvant chemotherapy in resected retroperitoneal sarcoma: evidence from a nationwide clinical oncology database and review of the literature. Surg Oncol. 2017;26(2):117-24.

12. Le Péchoux C, Musat E, Baey C, et al. Should adjuvant radiotherapy be administered in addition to front-line aggressive surgery (FAS) in patients with primary retroperitoneal sarcoma? Ann Oncol. 2013;24(3):832-7.

13. Zhou Z, McDade TP, Simons JP, et al. Surgery and radiotherapy for retroperitoneal and abdominal sarcoma: both necessary and sufficient. Arch Surg. 2010;145(5):426-31.

14. Peacock O, Patel S, Simpson JA, et al. A systematic review of populationbased studies examining outcomes in primary retroperitoneal sarcoma surgery. Surg Oncol. 2019;29:53-63.

15. Stahl JM, Corso CD, Park HS, et al. The effect of microscopic margin status on survival in adult retroperitoneal soft tissue sarcomas. Eur J Surg Oncol. 2017:43(1):168-74

16. Gronchi A, Lo Vullo S, Fiore M, et al. Aggressive surgical policies in a retrospectively reviewed single-institution case series of retroperitoneal soft tissue sarcoma patients. J Clin Oncol. 2009;27(1):24-30.

17. Gronchi A, Miceli R, Colombo C, et al. Frontline extended surgery is associated with improved survival in retroperitoneal low- to intermediategrade soft tissue sarcomas. Ann Oncol. 2012;23(4):1067-73.

18. Miura JT, Charlson J, Gamblin TC, et al. Impact of chemotherapy on survival in surgically resected retroperitoneal sarcoma. Eur J Surg Oncol. 2015;41(10):1386-92.

19. Nussbaum DP, Rushing CN, Lane WO, et al. Preoperative or postoperative radiotherapy versus surgery alone for retroperitoneal sarcoma: a case-control, propensity score-matched analysis of a nationwide clinical oncology database. Lancet Oncol. 2016;17(7):966-75.

20. Klooster B, Rajeev R, Chrabaszcz S, et al. Is long-term survival possible after margin-positive resection of retroperitoneal sarcoma (RPS)? J Surg Oncol. 2016;113(7):823-7.

21. Chouliaras K, Senehi R, Ethun CG, et al. Role of radiation therapy for retroperitoneal sarcomas: an eight-institution study from the US Sarcoma Collaborative. J Surg Oncol. 2019;120(7):1227-34.

22. Berger NG, Silva JP, Mogal $H$, et al. Overall survival after resection of retroperitoneal sarcoma at academic cancer centers versus community cancer centers: an analysis of the National Cancer Data Base. Surgery. 2018;163(2):318-23.

23. Choi AH, Barnholtz-Sloan JS, Kim JA. Effect of radiation therapy on survival in surgically resected retroperitoneal sarcoma: a propensity score-adjusted SEER analysis. Ann Oncol. 2012;23(9):2449-57.

24. ESMO/European Sarcoma Network Working Group. Soft tissue and visceral sarcomas: ESMO Clinical Practice Guidelines for diagnosis, treatment and follow-up. Ann Oncol. 2014;25 Suppl 3:iii102-iii112.

25. Almond LM, Gronchi A, Strauss D, et al. Neoadjuvant and adjuvant strategies in retroperitoneal sarcoma. Eur J Surg Oncol. 2018;44(5):571-9.

26. Zepeda E. Mario Cuellar Hubbé, Héctor Martínez Saíd Retroperitoneal soft tissue sarcoma. Bmc Cancer. 2007;7(Suppl 1):1-1.

27. Ballo MT, Zagars GK, Pollock RE, et al. Retroperitoneal soft tissue sarcoma: an analysis of radiation and surgical treatment. Int J Radiat Oncol Biol Phys. 2007;67(1):158-63.

28. Gilbeau L, Kantor G, Stoeckle E, et al. Surgical resection and radiotherapy for primary retroperitoneal soft tissue sarcoma. Radiother Oncol. 2002;65(3):137-43.

29. Canter RJ, Qin LX, Ferrone CR, et al. Why do patients with low-grade soft tissue sarcoma die? Ann Surg Oncol. 2008;15(12):3550-60.

30. Sargos P, Dejean C, de Figueiredo BH, et al. High-dose pre-operative helical tomotherapy (54 Gy) for retroperitoneal liposarcoma. Radiat Oncol. 2012;7:214.

31. Smith MJ, Ridgway PF, Catton CN, et al. Combined management of retroperitoneal sarcoma with dose intensification radiotherapy and resection: long-term results of a prospective trial. Radiother Oncol. 2014:110(1):165-71.

32. Bonvalot S, Gronchi A, Le Péchoux C, et al. Preoperative radiotherapy plus surgery versus surgery alone for patients with primary retroperitoneal sarcoma (EORTC-62092: STRASS): a multicentre, open-label, randomised, phase 3 trial. Lancet Oncol. 2020;21(10):1366-77.

33. Zlotecki RA, Katz TS, Morris CG, et al. Adjuvant radiation therapy for resectable retroperitoneal soft tissue sarcoma: the University of Florida experience. Am J Clin Oncol. 2005;28(3):310-6.

34. Pezner RD, Liu A, Chen YJ, et al. Full-dose adjuvant postoperative radiation therapy for retroperitoneal sarcomas. Am J Clin Oncol. 2011;34(5):511-6. 
35. Woll PJ, Reichardt P, Le Cesne A, et al. Adjuvant chemotherapy with doxorubicin, ifosfamide, and lenograstim for resected soft-tissue sarcoma (EORTC 62931): a multicentre randomised controlled trial. Lancet Oncol. 2012;13(10):1045-105.

\section{Publisher's Note}

Springer Nature remains neutral with regard to jurisdictional claims in published maps and institutional affiliations.
Ready to submit your research? Choose BMC and benefit from:

- fast, convenient online submission

- thorough peer review by experienced researchers in your field

- rapid publication on acceptance

- support for research data, including large and complex data types

- gold Open Access which fosters wider collaboration and increased citations

- maximum visibility for your research: over $100 \mathrm{M}$ website views per year

At BMC, research is always in progress.

Learn more biomedcentral.com/submissions 